J. Klin. Endokrinol. Stoffw. 2019 · 12:2-6 https://doi.org/10.1007/s41969-018-0053-4 Online publiziert: 16. Januar 2019 (c) Der/die Autor(en) 2019

CrossMark

\section{Thomas Stulnig}

Klinische Abteilung für Endokrinologie und Stoffwechsel, Klinik für Innere Medizin III, Medizinische Universität Wien, Wien, Österreich

\title{
Lysosomale Speicherkrankheiten im Erwachsenenalter
}

\section{Pathogenese lysosomaler Speicherkrankheiten}

Lysosomale Speicherkrankheiten (LSD) sind genetisch bedingte Erkrankungen, die durch Mutationen in kritischen Genen auf verschiedene molekulare Mechanismen schließlich zu einer Speicherung von Material in Lysosomen führen. Lysosomen sind die „Müllverbrennungsanlagen" der Zelle, die im Jahr 1955 von de Duve entdeckt wurden [1]. Es sind ca. $0,1-1,0 \mu \mathrm{m}$ im Durchmesser haltende Organellen, die von einer Lipidmembran umgeben sind und im Innern einen sauren $\mathrm{pH}$-Wert von 4-5 haben [2]. In ihnen wirkt eine Vielzahl von Enzymen, um das angelieferte Material abzubauen. Dazu gehören Proteinasen, Lipasen, Nukleasen, Glykosidasen, Sulfatasen und Phosphatasen, die die dazugehörigen Substrate spalten. Makromoleküle werden über Endo- und Phagozytose von außen aufgenommen, weiters intrazelluläre Moleküle durch Autophagozytose. Die Endosomen und Phagosomen verschmelzen mit primären Lysosomen aus dem Golgi-Apparat zu sekundären Lysosomen, in denen der Abbau stattfindet.

Bei LSD ist durch einen genetischen Defekt der Abbau von bestimmten Substanzen blockiert. LSD werden meist autosomal rezessiv vererbt, zwei Formen $\mathrm{X}$-chromosomal, wobei die Erkrankung z.T. auch bei Frauen, wenn auch milder, klinisch manifest werden kann. Eine lysosomale Speicherung von Glykogen in der Muskulatur wurde erstmals bei M. Pompe (Glykogenspeicherkrankheit II) als Krankheitsprinzip beschrieben. Durch die lysosomale Speicherung kommt es zu einer Größenzunahme der Zellen und zu einer progredienten Schä- digung von Organen. Da die Speicherung zumeist langsam erfolgt, sind Patienten mit LSD typischerweise bei Geburt unauffällig. Da die betroffenen Stoffwechselwege in verschiedenen Zelltypen und Organen unterschiedlich aktiv sind, ist auch die Speicherung und damit das klinische Bild sehr heterogen. Oft handelt es sich um Multisystemerkrankungen, da sich die Speicherung nicht auf einen Zelltyp beschränkt.

Charakterisiert werden LSD meist durch die Chemie des akkumulierten Substrats. So unterteilt man die LSD in Sphingolipidosen, Mukopolysaccharidosen, Oligosaccharidosen und Glykoproteinosen. Dazu kommen weitere LSD u.a. durch Defekte in integralen Membranproteinen. Die pathologische Speicherung von Material in Lysosomen führt zu Veränderungen nicht nur in der Zellstruktur, sondern auch in der Regulation des lysosomalen $\mathrm{pH}$ Werts, und beeinflusst weitere Prozesse wie die synaptische Freisetzung von Molekülen, die Endozytose, Vesikelreifung, Autophagie, Exozytose und zelluläre Kalzium-Homöostase [2]. Bei einer stetig zunehmenden Anzahl von LSD können viele dieser Veränderungen durch die spezifischen Therapien wie Enzymersatztherapie (ERT) und Substratreduktionstherapie (SRT) deutlich verbessert werden.

Entsprechend der Vielzahl an Molekülen, die für die Funktion von Lysosomen verantwortlich sind, ist auch die molekulare Pathogenese von LSD sehr heterogen [3]. Bei „klassischen“ LSD wie bei M. Niemann-Pick A/B (saurer Sphingomyelinase-Mangel, ASMD) liegt ein Enzymdefekt vor, der per se zu einer reduzierten Enzymaktivität führt. In ande- ren Fällen kann auch ein Enzym defekt sein, das eine lysosomale Hydrolase aktiviert. Dies ist z. B. der Fall beim GM2Aktivator-Mangel, einer Sonderform der GM2-Gangliosidose. Durch eine Mutation, die die Faltung des Enzyms im endoplasmatischen Retikulum stört, kann die fehlerhafte lysosomale Hydrolase nicht aus dem endoplasmatischen Retikulum (ER) in den Golgi-Apparat (und dann weiter ins Lysosom) transportiert werden, wie dies z. B. bei M. Gaucher der Fall ist. Bei der Galactosialidose kann durch ein defektes Cathepsin A der MultiEnzym-Komplex nicht gebildet werden, der zum Transport der lysosomalen Hydrolase aus dem ER in den Golgi-Apparat benötigt wird. Eine gestörte Glykosylierung im Golgi-Apparat kann zu einer verminderten Enzymaktivität der Hydrolasen im Lysosom führen. Transportstörungen verhindern, dass lysosomale Hydrolasen aus dem Golgi-Apparat in das Lysosom transportiert werden. In einer besonderen Form verhindert eine defekte Glykosylierung im Golgi-Apparat die Bindung der Hydrolasen an den Mannose-6-Phosphat-Rezeptor, der den Transport ins Lysosom sicherstellt. Dies führt bei der I-Zell-Erkrankung zu einer verminderten Enzymaktivität zahlreicher lysosomaler Hydrolasen und damit zur Speicherung verschiedener Substrate (Oligosaccharide, Mukopolysaccharide, Lipide). Einige LSD werde durch Defekte in integralen lysosomalen Membranproteinen hervorgerufen. Dazu gehören Transporterdefekte, wie bei der Cystinose, oder Defekte in anderen Schlüsselelementen der lysosomalen Funktion, wie dem „lysosomal-associated membrane protein-2“ (LAMP2), das der Danon-Erkrankung 
zugrunde liegt. Beim Multiplen Sulfatase-Mangel führen Mutationen im Sulfatase-modifizierenden Faktor-1(SUMF1)Gen zu einem defekten Ca-Formylglycin-generierenden Enzym (FGE), das das Cystein im aktiven Zentrum lysosomaler Sulfatasen nicht in Ca-Formylglycin umwandeln kann. Daraus resultieren inaktive Sulfatasen, die zwar über den Golgi-Apparat in die Lysosomen transportiert werden, dort aber keine Sulfatester abbauen können. Alle diese Defekte verschiedenster Natur resultieren damit in der lysosomalen Speicherung eines oder mehrerer Substrate.

Die Akkumulation von Substraten allein klärt aber nicht die Pathogenese von LSD. Fehlgefaltete Enzyme rufen endoplasmatischen Stress hervor und setzen eine „unfolded protein response“ in Gang. Durch den vermehrten Anfall an Substraten werden z.T. andere abbauende Stoffwechselwege beschritten, die z. T. zu inflammatorischen oder toxischen Metaboliten führen [4]. Die ver- änderte Zellmembranzusammensetzung bei Sphingolipidosen kann Signalwege verändern und die Zytokinese stören. Bestimmte Lipide können dazu noch die Kalziumfreisetzung aus dem endoplasmatischen Retikulum stören. Darüber hinaus spielen Veränderungen in der mitochondrialen Funktion einschließlich der Bildung freier Radikale sowie eine gestörte Endozytose und Autophagozytose eine wesentliche Rolle bei der Entstehung von LSD.

Das Fehlen des lysosomalen Abbaus führt zur Akkumulation nicht nur der Substrate, sondern auch der resultierenden Produkte. Der defekte lysosomale Abbau von Cholesterinestern und Triglyzeriden aus endozytierten Lipoproteinen bedingt damit ein Fehlen freier Fettsäuren und freien Cholesterins, die damit als Signalmoleküle für die Regulation der Lipid- und Cholesterinsynthese in der Leberzelle nicht mehr zur Verfügung stehen [5-7]. Es resultiert damit eine gesteigerte Lipogenese und Choles- terinproduktion, die über die vermehrte Sekretion von VLDL-Lipoproteinen den enzymatischen Defekt aggravieren. Durch fehlende Oxysterole wird auch der Kernrezeptor LXR nicht ausreichend aktiviert, sodass weniger Cholesterin aus den Zellen in das HDL hineingepumpt werden kann, wodurch der HDL-Cholesterinspiegel im Serum sinkt.

Genetisch bedingte Veränderungen lysosomaler Proteine können auch offensichtlich weitgehend unabhängig von ihrer enzymatischen Aktivität - auf ungeklärte Weise die Physiologie der Zelle stören. So stellen Mutationen im GBA1-Gen, das dem autosomal rezessiv vererbten M. Gaucher zugrunde liegt, in heterozygoter Form die häufigste genetische Ursache für den M. Parkinson dar.

\section{Klinik}

Wie erwähnt sind LSD klinisch sehr heterogene Erkrankungen. Die haupt-

Hier steht eine Anzeige. 
sächliche Organbeteiligung, die wiederum vom zugrunde liegenden Gendefekt abhängt, spielt dabei die wesentlichste Rolle. Eine Hepatosplenomegalie zeigt die Speicherung in diesen Organen an, Speicherung in Knochen (z. B. bei MPS) führt zu Knochdestruktionen, Skelettdysplasien oder Veränderungen der Kopfform. Knochenmarksbeteiligung (z.B. M. Gaucher) führt zu Blutbildveränderungen und Knocheninfarkten. An der Haut werden z.B. Angiokeratome (M. Fabry) oder Blutungen erkannt. Ähnlich kommen Beteiligungen von Nieren, zentralem und peripherem Nervensystem, des Herzens und der Muskulatur vor, sodass sehr unterschiedliche Krankheitsbilder entstehen.

Je nach Ausprägung des Enzymdefekts und gestörtem Abbauweg können LSD in jedem Lebensalter manifest werden, also von der Geburt bis ins hohe Erwachsenenalter (>70 Jahre) [8]. Schwerste Formen von LSD, die bereits im ersten Lebensmonat klinisch auffällig werden, sind unbehandelbar, wie z.B. der M. Gaucher Typ 2. Im Säuglingsalter werden z.B. die frühe Form des M. Pompe (Glykogenspeicherkrankheit Typ 2) mit schwerer Myopathie und insbesondere Kardiomyopathie manifest. Für diese und die infantile Form der LAL-D (früher: Wolman-Krankheit) gibt es bereits sehr erfolgreiche Enzymersatztherapien. Zahlreiche LSD werden bei Kindern und Jugendlichen diagnostiziert. Darunter fallen die $\mathrm{Mu}$ kopolysaccharidosen (MPS), die durch Kleinwuchs, Skelettdeformitäten und ggf. neurologische Störungen auffallen. Viele LSD stören die neurologische Entwicklung, wie die Krabbe- oder die TaySachs-Krankheit, und machen sich dementsprechend bemerkbar. Andere fallen durch Hepatosplenomegalie (M. Gaucher Typ 1 und 3, Niemann-Pick A/B/C), Angiokeratome und akrale Schmerzen (M. Fabry), Lungenbeteiligung (M. Gaucher Typ 3, Niemann-Pick B) oder Blutbildveränderungen (M. Gaucher Typ 1 und 3, Niemann-Pick B) auf. Myopathien der Achsen- und der proximalen Extremitätenmuskulatur stehen beim später beginnenden M. Pompe im Vordergrund. Im Erwachsenenalter werden typischerweise Erkrankungen mit
Hepatosplenomegalie und Blutbildveränderungen (M. Gaucher Typ 1 und 3, Niemann-Pick B und C, LAL-D), kardiovaskulären Erkrankungen (M. Fabry, Fucosidose, LAL-D), Nierenbeteiligung (Cystinose, M. Fabry) und psychiatrischen Symptomen (Metachromatische Leukodystrophie und späte Formen der GM2-Gangliosidose) manifest.

Selbst bei einzelnen Erkrankungen ist meist nicht ein Organ allein betroffen. Bei Patienten mit LAL-D entwickeln sich einerseits eine Leberzirrhose mit allen Folgen, andererseits auch Herz-Kreislauf-Erkrankungen, was die Speicherung in Hepatozyten und Endothelzellen, aber auch sekundäre Veränderungen durch die Hyperlipidämie abbildet. Bei M. Fabry findet sich ein komplexes Bild mit Speicherung in Nieren, Endothel, Augen (Cornea verticillata) und Herzmuskulatur (hypertrophe Kardiomyopathie). Bei M. Gaucher Typ 1 stehen die Hepatosplenomegalie, Thrombopenie und Knochenschmerzen bis hin zu den sog. Knochenkrisen im Vordergrund, daneben aber auch Allgemeinsymptome wie Blutungsneigung und Fatigue. Auch innerhalb einer Erkrankung haben damit nicht alle Patienten einen "typischen“ Verlauf, sondern unterscheiden sich im Spektrum und in der Intensität der Symptome.

Der wichtigste Punkt, um LSD zu diagnostizieren, liegt deshalb darin, an solche $\mathrm{zu}$ denken und entsprechende Tests zu veranlassen. Für einige LSD (z. B. M. Gaucher, M. Fabry, M. Pompe) wurden einfache Screeningtests auf Trockenblutbasis entwickelt, die kostenlos zur Verfügung gestellt werden. Alternativ können Patienten bei Verdacht an entsprechende Zentren zugewiesen werden. Jedenfalls müssen Patienten bei positivem Screeningtest an einem Zentrum vorgestellt werden, um die definitive Diagnostik und die Feststellung des Schweregrades aktueller Manifestationen der Erkrankungen zu veranlassen. Auch das Therapiemonitoring sollte an Stoffwechselzentren erfolgen.

\section{Therapie}

Durch ihre Anbindung an den Extrazellulärraum durch Endozytose gibt es die
Möglichkeit, große Moleküle wie Enzyme ins Lysosom zu bringen, um damit die Erkrankung zu therapieren. Die Enzyme für die Enzymersatztherapie (enzyme replacement therapy, ERT) werden dazu besonders mit Mannose- und Mannose-6-Phophat-Resten beladen, um die Aufnahme in die Lysosomen so effizient wie möglich zu gestalten. Die gegebenen Enzyme bauen dann im Lysosom die akkumulierten Substrate ab. Da Makrophagen eine besonders hohe endozytotische Aktivität aufweisen, ist die ERT bei M. Gaucher besonders wirksam [9]. Aber auch für M. Pompe, M. Fabry, LAL-D und eine Reihe von MPS wurden erfolgreich wirksame ERT entwickelt, und zahlreiche weitere stehen in verschiedenen Phasen der Entwicklung. Im Allgemeinen werden ERT ein- oder zweiwöchentlich intravenös verabreicht.

Da die ERT die Blut-Hirn-Schranke nicht passieren kann und damit nicht ins ZNS gelangt, werden bei systemischer Gabe zerebrale Manifestationen nicht behandelt. Kürzlich gab es einen Durchbruch in der Behandlung der neuronalen Ceroid-Lipofuscinose Typ 2 (CLN2, einer Form der Batten-Erkrankung) durch intraventrikuläre Gabe einer rekombinanten Tripeptidyl-Peptidase 1 (Cerliponase alfa) [10]. In Zukunft wird man also auch Therapien für andere LSD mit Affektion des ZNS erwarten können.

Ein neueres Therapieprinzip stellt die Substratdreduktionstherapie dar. Die Grundlage dafür ist, dass bei den meisten LSD eine kleine Restaktivität des Enzyms vorhanden ist, sodass das Substrat bei geringerer Produktion sehr wohl abgebaut werden könnte. Eine besonders wirksame und spezifische SRT ist für M. Gaucher entwickelt worden. Eliglustat führt ähnlich wie die ERT zu einer raschen Reduktion des Milz- und Lebervolumens sowie einer deutlichen Verbesserung der Blutbildveränderungen und anderer Biomarker [11, 12]. Auch auf die Konchenveränderungen hat Eliglustat einen sehr günstigen Einfluss, möglicherweise sogar noch besser als die ERT. Der große Unterschied für den Patienten besteht darin, dass SRT oral einzunehmen sind und damit die regelmäßigen Infusionen wegfallen. 
Ein weiteres Therapieprinzip, das bereits Eingang in die Klinik gefunden hat, ist die pharmakologische Chaperontherapie. Wie oben erwähnt, liegt bei einigen LSD das molekulare Problem darin, dass mutierte Proteine im ER falsch gefaltet werden und damit nicht in das Lysosom gelangen, sondern vorzeitig abgebaut werden. Chaperone sind Moleküle, die andere in ihrer korrekten Faltung unterstützen, pharmakologische Chaperone sind kleinmolekulare Substanzen, die eine solche Funktion haben. Sie binden oft an das aktive Zentrum des Enzyms und hemmen damit die Enzymaktivität. Solche pharmakologischen Chaperone müssen mit Pausen eingenommen werden, um einerseits die Produktion stabiler Enzyme und deren Transport ins Lysosom zu gewährleisten, andererseits in der Therapiepause auch deren enzymatische Aktivität zulassen. Mit Migalastat wurde unlängst ein wirksames pharmakologisches Chaperon in die Therapie des M. Fabry eingeführt [13]. Die Wirkung pharmakologischer Chaperone ist abhängig von der zugrunde liegenden Mutation, sodass z. B. für eine Therapie mit Migalastat nur ca. die Hälfte aller Mutationen im GLA-Gen in Frage kommen, während Enzyme, die andere Mutationen tragen, unbeeinflusst bleiben.

\section{Fazit für die Praxis \\ - Lysosomale Speichererkrankungen sind seltene Systemkrankheiten unterschiedlicher Pathogenese, klinischer Manifestationen und Schweregrade. \\ - Besonders (Hepato-)Splenomegalie, Blutbild-, Knochen-, Herz- und neuro- logische Veränderungen können auf diverse LSD hinweisen. \\ - Für einige LSD gibt es einfache Screeningtests, für die Diagnostik und Therapie sollten Patienten an Stoffwechselzentren mit Erfahrung in der Betreuung dieser Patienten zugewiesen werden. \\ - Eine Reihe von LSD kann wirksam mit Enzymersatztherapie, Substrat- reduktionstherapie oder pharma- kologischen Chaperonen behandelt werden.}

J. Klin. Endokrinol. Stoffw. 2019 · 12:2-6 https://doi.org/10.1007/s41969-018-0053-4

(c) Der/die Autor(en) 2019

\section{T. Stulnig}

\section{Lysosomale Speicherkrankheiten im Erwachsenenalter}

\section{Zusammenfassung}

Lysosomale Speicherkrankheiten (LSD) sind angeborene Erkrankungen, die zu einer Speicherung von nicht abbaubarem Material in den Lysosomen verschiedenster Zelltypen führen. Wenn auch einzelne LSD sehr selten sein können, so liegt die gesamte Inzidenz bei 1:8000 Lebendgeborenen. Trotz der prinzipiell gemeinsamen Pathogenese ist das klinische Bild sehr heterogen. So finden sich Vergrößerungen von Leber und/oder Milz wie beim M. Gaucher oder beim lysosomalen sauren Lipase-Mangel (LAL-D), Veränderungen am Skelettsystem wie bei den Mukopolysaccharidosen (MPS) und oft zentralnervöse Störungen wie bei verschiedenen Arten von Sphingolipidosen und MPS. Da die Speicherung oft Jahre benötigt, werden viele LSD erst im Erwachsenenalter klinisch manifest. Obwohl die
Defekte angeboren sind, gibt es für einige LSD wirksame Therapien. In der Enzymersatztherapie wird das fehlende Enzym in einer rekombinanten Form verabreicht. Die Substratreduktion reduziert die Produktion der sich ansammelnden Stoffe. Zuletzt war auch die pharmakologische ChaperonTherapie erfolgreich, die defekte Enzyme stabilisiert und damit deren vorzeitigen Abbau verhindert. Die Behandlung der zentralnervösen Veränderungen ist bis heute eine große Herausforderung.

\section{Schlüsselwörter}

Lysosomale Speicherkrankheiten · Seltene Krankheiten - Enzymersatztherapie - Substratreduktionstherapie. Pharmakologische Chaperone

\section{Lysosomal storage diseases in adults}

\section{Abstract}

Lysosomal storage diseases (LSD) are congenital disorders leading to the accumulation of undegradable material in lysosomes of different cell types. Even though individual LSD may be very rare, they sum up to an incidence of about 1:8000 live births. Despite the common principal pathogenesis, LSD are quite heterogeneous diseases. Some LSD, such as Gaucher's disease oder lysosomal acid lipase deficiency (LAL-D), present with enlargements of the liver and/or spleen, skeletal alterations as in mucopolysaccharidoses (MPS), and often central nervous changes as in various types of sphingolipidoses and MPS. Since accumulation of materials may take years, a number of LSD may not become clinically apparent before adulthood. Although LSD are genetic disorders, effective therapies exist for some of them. In enzyme replacement therapy (ERT), a recombinant form of the missing enzyme is given; substrate reduction therapy reduces the production of accumulating molecules. Recently, pharmacological chaperone therapy turned out to be successful as well by stabilizing defective enzyme and protecting them from premature degradation. Effective treatment of central nervous system alterations remains a major therapeutic challenge in many LSD.

\section{Keywords}

Lysosomal storage diseases · Orphan diseases Enzyme replacement therapy - Substrate reduction therapy - Pharmacological chaperones

\section{Korrespondenzadresse}

\section{Univ.-Prof. Dr. Thomas Stulnig}

Klinische Abteilung für Endokrinologie und Stoffwechsel, Klinik für Innere Medizin III, Medizinische Universität Wien Währinger Gürtel 18-20, 1090 Wien, Österreich thomas.stulnig@meduniwien.ac.at

Univ.-Prof. Dr. Thomas Stulnig Der Autor studierte Humanmedizin in Innsbruck und absolvierte die Ausbildung für Innere Medizin und die anschließende
Spezialisierung auf Stoffwechsel- und Hormonerkrankungen. Endokrinologe an der Medizinischen Universität Wien, wo er als Oberarzt tätig ist. Unter anderem leitet er dort die Ambulanz für angeborene Stoffwechselerkrankungen im Erwachsenenalter.

Funding. Open access funding provided by Medical University of Vienna. 


\section{Originalien}

\section{Einhaltung ethischer Richtlinien}

Interessenkonflikt. T. Stulnig erhielt Honorare, Forschungs- und Reisekostenunterstützung von Alexion, Biomarin, Sanofi-Genzyme, Shire.

Dieser Beitrag beinhaltet keine von den Autoren durchgeführten Studien an Menschen oder Tieren.

Open Access Dieser Artikel wird unter der Creative Commons Namensnennung 4.0 International Lizenz (http://creativecommons.org/licenses/by/4.0/deed. de) veröffentlicht, welche die Nutzung, Vervielfältigung, Bearbeitung, Verbreitung und Wiedergabe in jeglichem Medium und Format erlaubt, sofern Sie den/die ursprünglichen Autor(en) und die Quelle ordnungsgemäßnennen, einen Link zur Creative Commons Lizenz beifügen und angeben, ob Änderungen vorgenommen wurden.

\section{Literatur}

1. De Duve C (1955) Tissue fractionation studies. 6. Intracellular distribution patterns of enzymes in rat-liver tissue. Biochem J 60:604-617

2. Schultz ML, Tecedor L, Chang M, Davidson BL (2011) Clarifying lysosomal storage diseases. Trends Neurosci 34:401-410
3. Futerman AH, van Meer G (2004) The cell biology of lysosomal storage disorders. Nat Rev Mol Cell Biol 5:554-565

4. Thomas AS, Mehta A, Hughes DA (2014) Gaucher disease: haematological presentations and complications. Br J Haematol 165:427-440

5. Bernstein DL, Hulkova H, Bialer MG, Desnick RJ (2013) Cholesteryl ester storage disease: review of the findings in 135 reported patients with an underdiagnosed disease. J Hepatol 58:1230-1243 6. Rader DJ (2015) Lysosomal Acid Lipase Deficiency-A New Therapy for a Genetic Lipid Disease. NEngl J Med 373:1071-1073

7. Reiner Z, Guardamagna O, Nair D et al (2014) Lysosomal acid lipase deficiency-an underrecognized cause of dyslipidaemia and liver dysfunction. Atherosclerosis 235:21-30

8. Pastores GM, Hughes DA (2015) Non-neuronopathiclysosomal storagedisorders: Disease spectrum and treatments. Best Pract Res Clin Endocrinol Metab 29:173-182

9. Grabowski GA, Kacena K, Cole JA et al (2009) Doseresponse relationships for enzyme replacement therapy with imiglucerase/alglucerase in patients with Gaucher disease type 1.Genet Med 11:92-100

10. Schulz A, Ajayi T, Specchio N et al (2018) Study of Intraventricular cerliponase alfa for CLN2 disease. NEngl J Med 378:1898-1907

11. Mistry PK, Lukina E, Turkia BH et al (2015) Effect of oral eliglustat on splenomegaly in patients with Gaucher disease type 1: the ENGAGE randomized clinical trial. JAMA 313:695-706
12. Cox TM, Drelichman G, Cravo R et al (2017) Eliglustat maintains long-term clinical stability in patients with Gaucher disease type 1 stabilized on enzyme therapy. Blood 129:2375-2383

13. Germain DP, Hughes DA, Nicholls K et al (2016) Treatment of Fabry's Disease with the Pharmacologic Chaperone Migalastat. N Engl J Med 375:545-555

Hier steht eine Anzeige.

\section{Springer}

